\title{
Upscattering of ultracold neutrons from the polymer $\left[\mathrm{C}_{6} \mathrm{H}_{12}\right]_{n}$
}

\author{
E. I. Sharapov, ${ }^{1}$ C. L. Morris,,${ }^{2,}$ M. Makela,${ }^{2}$ A. Saunders, ${ }^{2}$ Evan R. Adamek,${ }^{3}$ L. J. Broussard,${ }^{2}$ C. B. Cude-Woods, ${ }^{3}$ \\ Deion E. Fellers, ${ }^{2}$ Peter Geltenbort, ${ }^{4}$ M. Hartl, ${ }^{2}$ S. I. Hasan, ${ }^{5}$ K. P. Hickerson, ${ }^{6}$ G. Hogan, ${ }^{2}$ A. T. Holley ${ }^{3}$ C. M. Lavelle, ${ }^{7}$ \\ Chen-Yu Liu, ${ }^{3}$ M. P. Mendenhall, ${ }^{6}$ J. Ortiz, ${ }^{2}$ R. W. Pattie, Jr., ${ }^{8}$ D. G. Phillips, II, ${ }^{8}$ J. Ramsey, ${ }^{2}$ D. J. Salvat, ${ }^{3}$ S. J. Seestrom, ${ }^{2}$ \\ E. Shaw, ${ }^{2}$ Sky Sjue, ${ }^{2}$ W. E. Sondheim, ${ }^{2}$ B. VornDick, ${ }^{8}$ Z. Wang, ${ }^{2}$ T. L. Womack, ${ }^{2}$ A. R. Young, ${ }^{8}$ and B. A. Zeck ${ }^{2,8}$ \\ ${ }^{1}$ Joint Institute for Nuclear Research, 141980 Dubna, Russia \\ ${ }^{2}$ Los Alamos National Laboratory, Los Alamos, New Mexico 87544, USA \\ ${ }^{3}$ Department of Physics, Indiana University, Indiana 47405-7105, USA \\ ${ }^{4}$ Institut Laue-Langevin, 38042 Grenoble Cedex 9, France \\ ${ }^{5}$ Department of Physics and Astronomy, University of Kentucky, Lexington, Kentucky 40506, USA \\ ${ }^{6}$ Kellogg Radiation Laboratory, California Institute of Technology, Pasadena, California 91125, USA \\ ${ }^{7}$ Applied Physics Laboratory, The Johns Hopkins University, Laurel, Maryland 20723, USA \\ ${ }^{8}$ Department of Physics, North Carolina State University, Raleigh, North Carolina 27695, USA
}

(Received 22 September 2013; published 6 December 2013)

\begin{abstract}
It is generally accepted that the main cause of ultracold neutron (UCN) losses in storage traps is upscattering to the thermal energy range by hydrogen adsorbed on the surface of the trap walls. However, the data on which this conclusion is based are poor and contradictory. Here we report a measurement, performed at the Los Alamos National Laboratory UCN source, of the average energy of the flux of upscattered neutrons after the interaction of UCN with hydrogen bound in the semicrystalline polymer PMP (trade name TPX), $\left[\mathrm{C}_{6} \mathrm{H}_{12}\right]_{n}$. Our analysis, performed with the MCNP code which applies the neutron-scattering law to UCN upscattered by bound hydrogen in semicrystalline polyethylene, $\left[\mathrm{C}_{2} \mathrm{H}_{4}\right]_{n}$, leads us to a flux average energy value of $26 \pm 3 \mathrm{meV}$, in contradiction to previously reported experimental values of 10 to $13 \mathrm{meV}$ and in agreement with the theoretical models of neutron heating implemented in MCNP.
\end{abstract}

DOI: 10.1103/PhysRevC.88.064605

PACS number(s): 78.70.Nx, 28.20.-v, 68.47.-b, 68.49.-h

Introduction. Ultracold neutrons (UCNs) are neutrons with kinetic energy below a critical value of $100 \mathrm{neV}$ (velocity $4.4 \mathrm{~m} / \mathrm{s}$ ). This corresponds to an average temperature of $1 \mathrm{mK}$, hence the technical term "ultracold". UCN are totally reflected from material surfaces at all angles of incidence and therefore can be confined in traps for time intervals of several hundred seconds, comparable to the neutron lifetime. Recent reviews [1,2] highlight the use of UCN in nuclear and particle physics, cosmology, and gravity. In particular, the importance of studies of the upscattering spectrum temperature and rates for neutron lifetime measurements is emphasized in Ref. [3]. Authors of Ref. [4] discuss using UCN upscattering techniques in solid-state and surface studies that will be made possible by more powerful next-generation UCN sources. For all these studies a better understanding of UCN interactions, especially upscattering, at surfaces of different materials continues to be of importance in view of existing inconsistencies in data, as detailed in Ref. [4]. During transport in neutron guides or storage in material traps, UCN can be excited above the critical energy by absorbing energy from thermal excitation of the surface materials and leaving the confinement space. It is presently believed that the main reason for UCN heating in traps is inelastic scattering from the hydrogen molecules on the material surfaces. Indeed, by the nuclear reaction analysis method [5] with a ${ }^{15} \mathrm{~N}$ beam impinging on unbaked copper samples, the areal density of the surface hydrogen was

*Corresponding author: cmorris@lanl.gov determined to be $2 \times 10^{16} \mathrm{H} / \mathrm{cm}^{2}$ and the hydrogen-containing layer was estimated to be $3.0 \mathrm{~nm}$ thick.

Hydrogen has one of the largest inelastic scattering cross sections of all elements. It is most convenient to study it in polyethylene, $\left[\mathrm{C}_{2} \mathrm{H}_{4}\right]_{n}$, in which the hydrogen inelastic cross section increases as the inverse velocity law to the value of $2053 \pm 40 \mathrm{~b}$ [6] at a velocity of $4.0 \mathrm{~m} / \mathrm{s}$. The authors of Refs. $[7,8]$ used a $100-\mu$ m-thick polyethylene (PE) sample inside a UCN trap and $\sim 4 \pi{ }^{3} \mathrm{He}$ neutron detectors of a different gas pressure to observe the upscattered neutron flux and have determined its average energy to be $10-13 \mathrm{meV}$ (the corresponding velocity $\sim 1600 \mathrm{~m} / \mathrm{s}$ ). This is about half of the value expected from the known phonon frequency spectrum of hydrogen in PE and has triggered further efforts to find possible channels of the UCN escaping from traps. The authors of Ref. [9], using the neutron activation method, searched for lower energy upscattering of UCN from a Be surface. A possibility of scattering in the range of 15 to $300 \mathrm{~m} / \mathrm{s}$ was convincingly ruled out. Also, so-called "weak heating" with UCN energy changes $\simeq 10 \mathrm{neV}$ has been reported [10], with a probability estimated to be $\sim 10^{-7}$ per the collision with copper, which is a rather low value. In this paper, we study UCN upscattering into the thermal energy range in polymethylpentene (PMP), $\left[\mathrm{C}_{6} \mathrm{H}_{12}\right]_{n}$, which, owing to its low density $\left(0.83 \mathrm{~g} / \mathrm{cm}^{3}\right.$, compared to $0.92-0.97 \mathrm{~g} / \mathrm{cm}^{3}$ for $\left.\mathrm{PE}\right)$, is a material with negative, practically nonreflective optical potential $U_{F}$ even smaller than that of PE, $\left[\mathrm{C}_{2} \mathrm{H}_{4}\right]_{n}$. The $U_{F}$ 's are approximately -7.8 and $-8.9 \mathrm{nV}$ for PMP and PE, respectively. Our PMP (trade name TPX, Mitsui Chemicals, Inc., for the atactic poly[4-methyl-1-pentene] material) was 


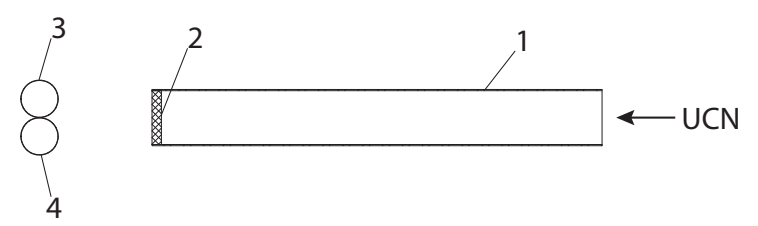

FIG. 1. Scheme of a section of the neutron guide and the drift-tube-detector placement for measuring upscattering of ultracold neutrons: 1, the neuron guide; 2 , the sample; 3,4 , the neutron detectors.

a tetragonal of Form I semicrystalline sample with an x-ray diffraction spectrum similar to that of the undrawn sample in Ref. [11], as was evidenced by our own x-ray diffraction data.

Measurements. The measurements have been performed at the Los Alamos National Laboratory solid-deuterium ultracold neutron source driven by the $800-\mathrm{MeV}, 5.8-\mu \mathrm{A}$ average proton beam provided by the Los Alamos Neutron Science Center (LANSCE) linear accelerator. The source is described in detail in a recent publication by Saunders et al. [12]. A schematic of the experimental geometry is shown in Fig. 1. A 7.62-cm-diameter stainless steel UCN guide tube (1) was connected to the main UCN guide through the section containing a flange with a zirconium foil embedded in the 6-T field of a superconducting solenoid magnet. This $\mathrm{Zr}$ foil separates the UCN source vacuum system from the external guide. The magnetic field accelerates half of the neutrons above the foil's critical energy, allowing the high-field-seeking beam to exit the UCN source, reducing losses owing to transmission through the foil. The 500- $\mu$ m-thick PMP sample (2), with the same diameter as the inside of the tube, was installed at the end of the shown section. The UCN density in the tube was $\simeq 1.0 \mathrm{UCN} / \mathrm{cm}^{3}$, and the average velocity of the UCN flux was $\simeq 4 \mathrm{~m} / \mathrm{s}$. Two 5 -cm-diameter, 30 -cm-long drift-tube ${ }^{3} \mathrm{He}$ neutron detectors (3) and (4) were installed perpendicular to the guide axis and symmetrically above and below it, as shown in Fig. 1, to provide equal fluxes for both detectors. The construction, gas filling, and performance of detectors are described in Ref. [13]. The partial ${ }^{3} \mathrm{He}$ pressure was $180 \mathrm{kPa}$ in one detector and $20 \mathrm{kPa}$ in the other one. Data were accumulated for $300 \mathrm{~s}$ under steady beam conditions. The upscattered neutron rates were significantly above the background, which was mostly attributable to cosmogenic thermal neutrons. The background associated with the proton beam was eliminated using time gates on the analog-to-digital converters to reject events during the beam pulses. The neutron rates for the analysis were calculated by integrating the measured pulse-height spectra from both detectors. The ratio $R=N_{1.8} / N_{0.2}$ of integrated rates was compared with calculations to deduce the average energy $\langle E\rangle$ of the upscattered flux. Data were also taken with a 6.3-mm-thick PE slab inserted between detectors and the end of the UCN guide to thermalize completely the upscattered neutrons and to compare the result with calculations.

Analysis. In principle, if the scattered flux has Maxwellian shape, the flux average energy $\langle E\rangle$ can be easily estimated. The efficiencies $\epsilon$ of the ${ }^{3} \mathrm{He}$ detectors for neutrons with energy $E$ crossing the tubes along the same track $\ell$ are

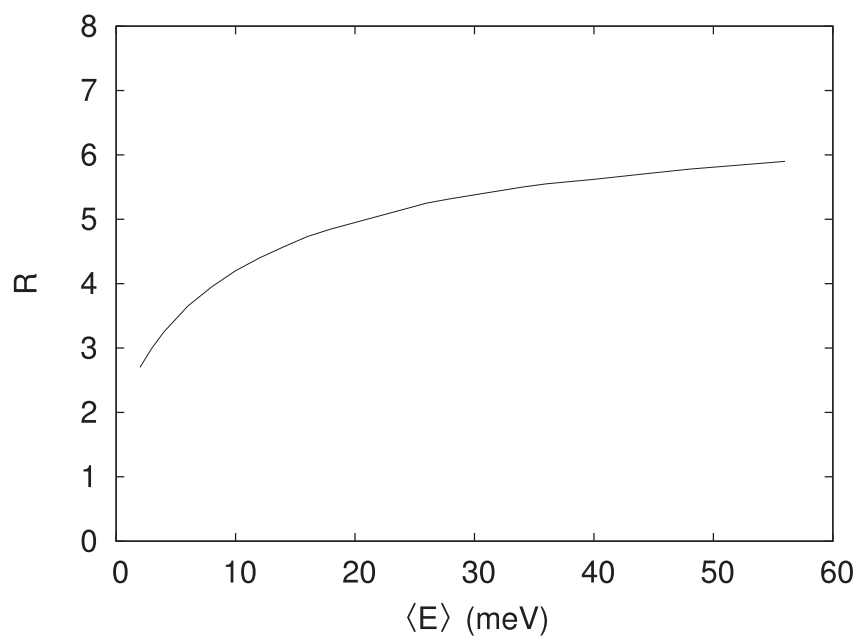

FIG. 2. The calculated ratio $R$ of the Los Alamos National Laboratory drift tubes (with the 180 and $20 \mathrm{kPa}$ of ${ }^{3} \mathrm{He}$ partial pressure) efficiencies versus the average energy $\langle E\rangle$ of the Maxwellian scattered flux. It would give the result for $\langle E\rangle$ from measured $R$ if the assumption of the Maxwellian shape for the neutron flux is valid. As explained in the text, we do not use this assumtion in analyzing our data.

different because $\epsilon=1-\exp \left\{-N\left({ }^{3} \mathrm{He}\right) \ell \sigma(E)\right\}$, while the helium-3 atomic densities $N\left({ }^{3} \mathrm{He}\right)$ are different for the 180 - and 20-kPa detectors $(\sigma(E)$ is a well-known cross section for the ${ }^{3} \mathrm{He}(n, p) T$ reaction). We calculated efficiencies for several Maxwellian spectra with different $\langle E\rangle$ using the code MCNP5 [14] to follow all neutron trajectories crossing our detectors in the geometry of Fig. 1. A calculation of $R$ vs $\langle E\rangle$ (shown in Fig. 2) provides then the average energy of the Maxwellian upscattered spectrum using the measured value of $R$. However, a Maxwellian shape for the upscattered flux is not expected from the theory of neutron inelastic scattering by a bound hydrogen.

For UCN upscattering, a simple analytical formula for the isotropic differential cross section in the one-phonon incoherent approximation,

$$
\frac{d \sigma_{\mathrm{up}}}{d E}=\sigma_{\mathrm{b}} \sqrt{\frac{E}{E_{i}}}\left(e^{E / k T}-1\right)^{-1} \frac{g(E)}{\mathrm{A}} e^{-2 W},
$$

demonstrates clearly that the upscattering spectrum is not Maxwellian. This equation, written initially in terms of the neutron wave-vector variable, was obtained by Placzek and Van Hove [15]. Here $\sigma_{\mathrm{b}}=4 \pi b^{2}$ is the cross section for bound nuclei with mass number $A, E_{i}$ is the initial UCN energy, $E$ is the energy after upscattering, $g(E)$ is the generalized (amplitude weighted) phonon density of states in the material under study, and the last exponent is the material- and temperature-dependent Debye-Waller factor. At a temperature of $300 \mathrm{~K}$, multiphonon contributions are also important [16], and a full upscattering spectrum is often calculated with the use of MCNP and its models for the neutron-scattering law $S(\alpha, \beta)$ [17] ( $\alpha$ and $\beta$ are the reduced neutron momentum and energy transfers, respectively), which takes partial account of multiphonon processes. The issue of different theoretical approaches to multiphonon scattering is of interest itself but we 
leave it out of the present report because our experimental data cannot distinguish between them. A more recent model of the generalized density of states of hydrogen in PE is provided by Barrera et al. [18]. It was validated recently by neutron inelastic measurements [19] for the high-density PE. Using these data and the deduced $S(\alpha, \beta)$, one of the authors (C.M.L.) created $77 \mathrm{~K}$ and $293 \mathrm{~K}$ scattering kernels, represented as tables of the double differential scattering cross sections for modeling the neutron transport in PE. Although such data are absent for the polymer PMP, we believe that its neutron-scattering law is similar to the $S(\alpha, \beta)$ of PE and one can use PE as a model for UCN upscattering from PMP. Indeed, the known experimental infrared spectra are similar for PMP [20] and PE [21] and the same is valid for numerous theoretical calculations, as referenced, for example, in Ref. [22]. The presence of the methyl groups in the side branches of the PMP molecular chain can additionally influence the low-energy range of PMP phonon density of states, not accessible in infrared and Raman spectroscopy, and we address this issue in the concluding paragraph.

Therefore, with the new PE kernels added to the MCNP thermal energy data, we modeled the scattered flux from our PMP sample in the experimental geometry of Fig. 1 with an initial neutron beam energy $E_{\text {in }}=100 \mathrm{neV}$. The results are shown in Fig. 3. The shape of the directly upscattered flux (black squares) is clearly not Maxwellian and was found to be independent of the initial neutron energy and the placement of detectors. The spectrum of the fully moderated (by the additional 6.3-mm-thick PE slab placed between the sample and detectors) flux is Maxwellian. Using these spectral shapes, we modeled the ${ }^{3} \mathrm{He}(n, p) T$ reaction rates in the two detectors after the UCN scattering using the $293 \mathrm{~K}$ kernel. The results of modeling together with the results of measurements are presented in Table I for comparison.

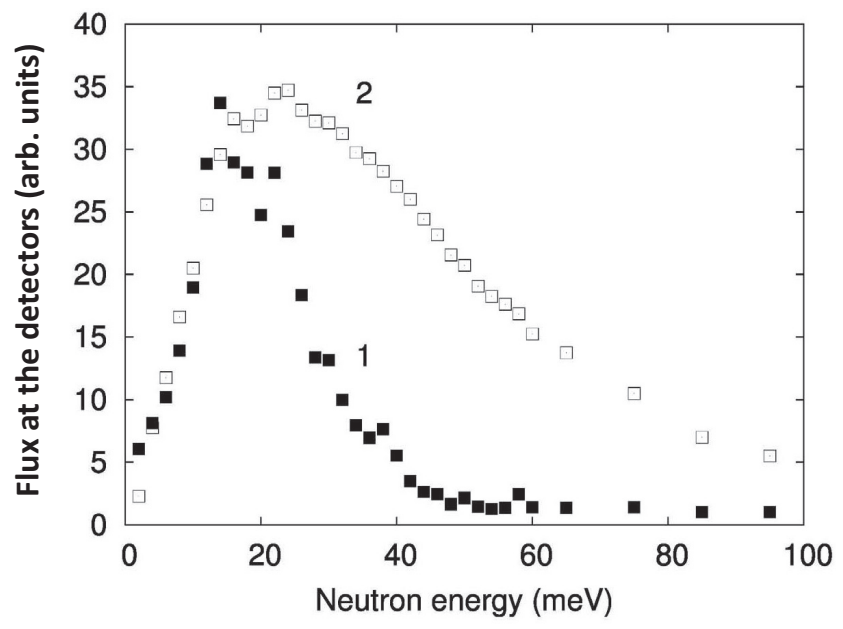

FIG. 3. The calculated energy spectra of the neutron flux scattered from the PE sample, $500 \mu \mathrm{m}$ thick, $E_{\text {in }}=100 \mathrm{neV}$ (curve 1), and of the fully moderated flux (curve 2) after "filtering" this flux through the 6-mm PE slab. The spectra are obtained by MCNP modeling with the $293 \mathrm{~K} S(\alpha, \beta)$ scattering law. The shape of spectrum 1 is not Maxwellian, it has an average energy of $26 \pm 3 \mathrm{meV}$. Spectrum 2 is a Maxwellian with an average energy of $53 \pm 4 \mathrm{meV}$.
TABLE I. Experimental results in comparison with the MCNP modeling. $N(1.8)$ and $N(0.2)$ represent count rates taken during the 300 -s run with detectors of the 180 - and $20-\mathrm{kPa}^{3} \mathrm{He}$ pressure. $R(\exp )$ and $R$ (MCNP) represent their ratios.

\begin{tabular}{lcccc}
\hline \hline Neutron spectrum & $N(1.8)$ & $N(0.2)$ & $R(\exp )$ & $R(\mathrm{MCNP})$ \\
\hline Upscattered & 71159 & 14943 & $4.76 \pm 0.07$ & 4.67 \\
Moderated & 32360 & 5264 & $6.14 \pm 0.09$ & 5.90 \\
Double ratio & & & $0.77 \pm 0.02$ & 0.79 \\
\hline \hline
\end{tabular}

Conclusions. As shown in Table I, the measurements on the PMP sample and MCNP modeling with the use of the PE $293 \mathrm{~K}$ kernel based on theoretical $S(\alpha, \beta)$ scattering law agree rather well. In the mode, the non-Maxwellian scattered spectrum for a thin sample has the average energy value of $26 \pm 3 \mathrm{meV}$. Therefore, we conclude that, after the initial UCN energy, the spectrum of neutrons, scattered in one or only few interactions, has the expected non-Maxwellian shape and the average energy $\langle E\rangle=26 \pm 3 \mathrm{meV}$. At the same time, the fully moderated flux spectrum has Maxwellian shape with the average energy of $53 \pm 4 \mathrm{meV}$ at room temperature. Our conclusion contradicts the value $\langle E\rangle=10-13 \mathrm{meV}[7,8]$ obtained in essentially the same kind of experiment, although with a different ${ }^{3} \mathrm{He}$ detector, with a PE sample, and by the analysis in a frame of Maxwellian approximation. This situation led us to reanalyze data $[7,8]$ using MCNP with the input describing geometry and conditions of the measurement [8]. The result is shown in Fig. 4, where the solid squares are experimental data of Ref. [8] and curves 1 and 2 have been

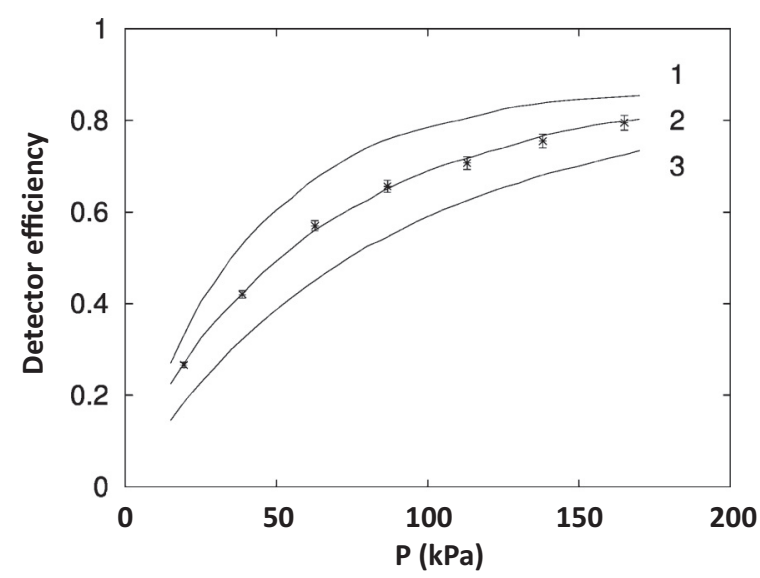

FIG. 4. Reanalysis of the Stoika and Strelkov [8] PE experiment. Experimental data [8] for their neutron detector efficiencies as a fuction of the ${ }^{3} \mathrm{He}$ pressure are shown as crosses. Curves 1 and 2 show our MCNP modeling of their detector efficiencies, which were performed using the PE scattering law $S(\alpha, \beta)$ kernels at $77 \mathrm{~K}$ and $293 \mathrm{~K}$. Curve 1 corresponds to the non-Maxwellian upscattered spectrum with $\langle E\rangle=11 \mathrm{meV}$, for getting which we resorted to in the $77 \mathrm{~K}$ kernel. Curve 2 corresponds to the $293 \mathrm{~K}$ spectrum with $\langle E\rangle=26 \mathrm{meV}$. Curve 3 for the $293 \mathrm{~K}$ Maxwellian spectrum is shown, following Ref. [8] for comparison. From the agreement of data [8] with curve 2 we deduce the average energy of $26 \mathrm{meV}$ for their PE experiment, which is the same as the result of our PMP experiment. 
obtained by MCNP with the scattering law $S(\alpha, \beta)$ kernels at $77 \mathrm{~K}$ and $293 \mathrm{~K}$ to produce non-Maxwellian theoretical spectra with $\langle E\rangle=11 \mathrm{meV}$ and $\langle E\rangle=26 \mathrm{meV}$, respectively. Curve 3 for the $293 \mathrm{~K}$ Maxwellian spectrum is shown for comparison. From agreement of the experimental data with curve 2 we claim the average energy of $26 \mathrm{meV}$ in the PE experiment of Stoika and Strelkov [8], the same average energy as in our PMP experiment modeled with the neutron-scattering law $S(\alpha, \beta)$ for PE.

This comparison of two experiments allows us to conclude that, the phonon densities of states in PE and PMP are similar.
Finding possible low-energy distinctions between them would require dedicated UCN and thermal neutron inelastic scattering measurements.

Acknowledgments. We thank L. Daemen for help in acquiring $\mathrm{x}$-ray diffraction data for our samples. This work was performed under the auspices of the U.S. Department of Energy under Contract No. DE-AC52-06NA25396. Author D.J.S. is supported by the DOE Office of Science Graduate Fellowship Program, made possible in part by the American Recovery and Reinvestment Act of 2009, administered by ORISE-ORAU under Contract No. DE-AC05-06OR23100.
[1] W. Michael Snow, Phys. Today 66(3), 50 (2013).

[2] S. K. Lamoreaux and R. Golub, J. Phys. G: Nucl. Part. Phys. 36, 104002 (2009).

[3] R. Picker et al., Nucl. Instrum. Methods Phys. Res., Sect. A 611, 297 (2009).

[4] E. Korobkina, R. Golub, J. Butterworth, P. Geltenbort, and S. Arzumanov, Phys. Rev. B 70, 035409 (2004).

[5] W. A. Lanford and R. Golub, Phys. Rev. Lett. 39, 1509 (1977).

[6] Yu. N. Pokotilovski, M. I. Novopoltsev, P. Geltenbort, and T. Brenner, Instrum. Exp. Tech. 54, 16 (2011).

[7] A. D. Stoika, A. V. Strelkov, and M. Hetzelt, Z. Phys. B 29, 349 (1978).

[8] A. D. Stoika and A. V. Strelkov, Report P3-11593, JINR, Dubna (1978).

[9] Al. Yu. Muzhichka, Yu. N. Pokotilovski, and P. Geltenbort, J. Exp. Theor. Phys. 88, 79 (1999).

[10] A. V. Strelkov, V. V. Nesvizhevsky, P. Geltenbort et al., Nucl. Instrum. Methods Phys. Res., Sect. A 440, 695 (2000).

[11] Tianbei He and Roger S. Porter, Polymer 28, 1321 (1987).
[12] A. Saunders, M. Makela, Y. Bagdasarova et al., Rev. Sci. Instrum. 84, 013304 (2013).

[13] Zhehui Wang, C. L. Morris, M. Makela et al., Nucl. Instrum. Methods Phys. Res., Sect. A 605, 430 (2009).

[14] X-5-Monte-Carlo-team, Los Alamos National Laboratory Report No. LA-UR-03-198, 2003.

[15] G. Placzek and L. Van Hove, Phys. Rev. 93, 1207 (1954).

[16] T. Kitagawa and T. Miyazawa, J. Polym. Sci., Part B: Polym. Lett. 6, 83 (1968).

[17] R. E. MacFarlane, in Handbook of Neutron Engineering (Springer Science and Business Media, USA, 2010), pp. 189-277.

[18] G. D. Barrera, S. F. Parker, A. J. Ramirez-Cuesta, and P. C. H. Mitchell, Macromolecules 39, 2683 (2006).

[19] C. M. Lavelle, C.-Y. Liu, and M. B. Stone, Nucl. Instrum. Methods Phys. Res., Sect. A 711, 166 (2013).

[20] SIGMA-ALDRICH LLC, http://www.sigmaaldrich.com/ spectra/rair/RAIR003202.PDF.

[21] S. Krimm, C. Y. Liang, and G. B. B. Sutherland, J. Chem. Phys. A 25, 549 (1956).

[22] A. Kumar et al., Macromol. Symp. 277, 51 (2009). 